\title{
Existence and Decay Estimate of Global Solutions to Systems of Nonlinear Wave Equations with Damping and Source Terms
}

\author{
Yaojun Ye \\ Department of Mathematics and Information Science, Zhejiang University of Science and Technology, Hangzhou 310023, China \\ Correspondence should be addressed to Yaojun Ye; yeyaojun@zust.edu.cn
}

Received 30 April 2013; Revised 1 September 2013; Accepted 2 September 2013

Academic Editor: T. Raja Sekhar

Copyright (C) 2013 Yaojun Ye. This is an open access article distributed under the Creative Commons Attribution License, which permits unrestricted use, distribution, and reproduction in any medium, provided the original work is properly cited.

The initial-boundary value problem for a class of nonlinear wave equations system in bounded domain is studied. The existence of global solutions for this problem is proved by constructing a stable set and obtain the asymptotic stability of global solutions through the use of a difference inequality.

\section{Introduction}

In this paper, we are concerned with the global solvability and decay stabilization for the following nonlinear wave equations system:

$$
\begin{aligned}
u_{t t} & -\operatorname{div}\left(|\nabla u|^{p-2} \nabla u\right)+\left|u_{t}\right|^{q-2} u_{t}-\Delta u_{t} \\
& =|v|^{r+2}|u|^{r} u, \quad(x, t) \in \Omega \times R^{+}, \\
v_{t t} & -\operatorname{div}\left(|\nabla v|^{p-2} \nabla v\right)+\left|v_{t}\right|^{q-2} v_{t}-\Delta v_{t} \\
& =|u|^{r+2}|v|^{r} v, \quad(x, t) \in \Omega \times R^{+}
\end{aligned}
$$

with the initial-boundary value conditions

$$
\begin{array}{r}
u(x, 0)=u_{0}(x) \in W_{0}^{1, p}(\Omega), \quad u_{t}(x, 0)=u_{1}(x) \in L^{2}(\Omega) \\
x \in \Omega, \\
(3) \\
v(x, 0)=v_{0}(x) \in W_{0}^{1, p}(\Omega), \quad v_{t}(x, 0)=v_{1}(x) \in L^{2}(\Omega) \\
x \in \Omega, \\
u(x, t)=0, \quad v(x, t)=0, \quad(x, t) \in \partial \Omega \times R^{+}, \quad
\end{array}
$$

where $\Omega$ is a bounded open domain in $R^{n}$ with a smooth boundary $\partial \Omega, p, q \geq 2, r>0$ and $p<2(r+2) \leq n p /(n-p)$ for $n \geq p$ and $p<2(r+2)<+\infty$ for $n<p$.
When $p=2$, Medeiros and Miranda [1] proved the existence and uniqueness of global weak solutions. Cavalcanti et al. in [2-4] considered the asymptotic behavior for wave equation and an analogous hyperbolic-parabolic system with boundary damping and boundary source term. In paper [5, 6], the authors dealt with the existence, uniform decay rates, and blowup for solutions of systems of nonlinear wave equations with damping and source terms.

Rammaha and Wilstein [7] and Yang [8] are concerned with the initial boundary value problem for a class of quasilinear evolution equations with nonlinear damping and source terms. Under appropriate conditions, by a Galerkin approximation scheme combined with the potential well method, they proved the existence and asymptotic behavior of global weak solutions when $m<p$, where $m \geq 0$ and $p$ are, respectively, the growth orders of the nonlinear strain terms and the source term.

Ono [9] considers the following initial-boundary value problem for nonlinear wave equations with nonlinear dissipative terms:

$$
\begin{array}{r}
u_{t t}-\Delta u+\delta_{1} u_{t}+\delta_{2}\left|u_{t}\right|^{\beta} u_{t}-\delta_{3} \Delta u_{t}=|u|^{\alpha} u, \\
(x, t) \in \Omega \times R^{+}, \\
u(x, 0)=u_{0}(x), \quad u_{t}(x, 0)=u_{1}(x), \quad x \in \Omega, \\
u(x, t)=0, \quad x \in \partial \Omega, t \geq 0,
\end{array}
$$


where $\delta_{i} \geq 0, i=1,2,3$, and $\alpha, \beta>0$ are constants. The author mainly investigates on the blowup phenomenon to problem (6). On the other hand, in the case of $\delta_{1}+\delta_{2}+\delta_{3}>0$, he shows that the problem (6) admits a unique global solution, and its energy has some decay properties under some assumptions on $u_{0}$ and initial energy $E(0) \equiv E\left(u_{0}, u_{1}\right)$. In particular, when $\delta_{2}>0$ and $\delta_{1}+\delta_{3}>0$ in (6), the energy $E(t) \equiv$ $E\left(u(t), u_{t}(t)\right)$ has some polynomial and exponential decay rates, respectively. tion

For the following strongly damped nonlinear wave equa-

$$
u_{t t}-\Delta u_{t}-\Delta u+f\left(u_{t}\right)+g(u)=h,
$$

Dell'Oro and Pata [10] obtain the long-time behavior of the related solution semigroup, which is shown to possess the global attractor in the natural weak energy space. In addition, the existence of global and local solutions, decay estimates, and blowup for solutions of nonlinear wave equation with source and damping terms and exponential nonlinearities are studied in [11-14].

In this paper, we prove the global existence for the problem (1)-(5) by applying the potential well theory introduced by Sattinger [15] and Payne and Sattinger [16]. Meanwhile, we obtain the asymptotic stabilization of global solutions by using a difference inequality [17].

For simplicity of notations, hereafter we denote by $\|\cdot\|_{p}$ the norm of $L^{p}(\Omega) ;\|\cdot\|$ denotes $L^{2}(\Omega)$ norm, and we write equivalent norm $\|\cdot \nabla\|_{p}$ instead of $W_{0}^{1, p}(\Omega)$ norm $\|\cdot\|_{W_{0}^{1, p}(\Omega)}$. Moreover, $C$ denotes various positive constants depending on the known constants and may be different at each appearance.

\section{Local Existence}

In this section, we investigate the local existence and uniqueness of the solutions of the problem (1)-(5). For this purpose, we list up two useful lemmas which will be used later and give the definition of weak solutions.

Lemma 1. Let $u \in W_{0}^{1, p}(\Omega)$, then $u \in L^{s}(\Omega)$; and the inequality $\|u\|_{s} \leq C\|u\|_{W_{0}^{1, p}(\Omega)}$ holds with a constant $C>0$ depending on $\Omega$, $p$, and $s$, provided that $2 \leq s<+\infty, 2 \leq n \leq p$ and $2 \leq s \leq n p /(n-p), 2<p<n$.

Lemma 2 (Young inequality). Let $a, b \geq 0$ and $1 / p+1 / q=1$ for $1<p, q<+\infty$; then one has the inequality

$$
a b \leq \delta a^{p}+C(\delta) b^{q},
$$

where $\delta>0$ is an arbitrary constant, and $C(\delta)$ is a positive constant depending on $\delta$.

Definition 3. A pair of functions $(u, v)$ is said to be a weak solution of (1)-(5) on $[0, T]$ if $u, v \in C\left([0, T], W_{0}^{1, p}(\Omega)\right)$, $u_{t}, v_{t} \in C\left([0, T], L^{2}(\Omega)\right),[u(0), v(0)]=\left[u_{0}, v_{0}\right] \in W_{0}^{1, p}(\Omega) \times$ $W_{0}^{1, p}(\Omega),\left[u_{t}(0), v_{t}(0)\right]=\left[u_{1}, v_{1}\right] \in L^{2}(\Omega) \times L^{2}(\Omega)$, and $[u, v]$ satisfies

$$
\begin{aligned}
& \left\langle u_{t}(t), \phi\right\rangle_{L^{2}(\Omega)}-\left\langle u_{1}, \phi\right\rangle_{L^{2}(\Omega)} \\
& \quad+\int_{0}^{t}\left\langle\left(|\nabla u|^{p-2} \nabla u\right), \nabla \phi\right\rangle_{L^{2}(\Omega)} d \tau \\
& \quad+\int_{0}^{t}\left\langle\left|u_{t}\right|^{q-2} u_{t}, \phi\right\rangle_{L^{2}(\Omega)} d \tau+\int_{0}^{t}\left\langle\nabla u_{t}, \nabla \phi\right\rangle_{L^{2}(\Omega)} \\
& =\int_{0}^{t}\left\langle|v|^{r+2}|u|^{r} u, \phi\right\rangle_{L^{2}(\Omega)} d \tau, \\
& \left\langle v_{t}(t), \psi\right\rangle_{L^{2}(\Omega)}-\left\langle v_{1}, \psi\right\rangle_{L^{2}(\Omega)} \\
& \quad+\int_{0}^{t}\left\langle\left(|\nabla v|^{p-2} \nabla v\right), \nabla \psi\right\rangle_{L^{2}(\Omega)} d \tau \\
& \quad+\int_{0}^{t}\left\langle\left|v_{t}\right|^{q-2} v_{t}, \psi\right\rangle_{L^{2}(\Omega)} d \tau+\int_{0}^{t}\left\langle\nabla v_{t}, \nabla \psi\right\rangle_{L^{2}(\Omega)} \\
& =\int_{0}^{t}\left\langle|u|^{r+2}|v|^{r} v, \psi\right\rangle_{L^{2}(\Omega)} d \tau,
\end{aligned}
$$

for all test functions $\phi, \psi \in W_{0}^{1, p}(\Omega)$ and for almost all $t \in$ $[0, T]$.

The local existence and uniqueness of solutions for problem (1)-(5) can be proved through the use of Galerkin method. The result reads as follows.

Theorem 4 (local solution). Supposed that $\left[u_{0}, v_{0}\right] \in$ $W_{0}^{1, p}(\Omega) \times W_{0}^{1, p}(\Omega),\left[u_{1}, v_{1}\right] \in L^{2}(\Omega) \times L^{2}(\Omega)$, and $p<2(r+$ $2) \leq n p /(n-p)$ if $n \geq p$ and $p<2(r+2)<+\infty$ for $n<p$, then there exists $T>0$ such that the problem (1)-(5) has a unique local solution $[u(t), v(t)]$ satisfying

$$
\begin{gathered}
{[u, v] \in L^{\infty}\left([0, T) ; W_{0}^{1, p}(\Omega) \times W_{0}^{1, p}(\Omega)\right) ;} \\
{\left[u_{t}, v_{t}\right] \in L^{\infty}\left([0, T) ; L^{2}(\Omega) \times L^{2}(\Omega)\right),} \\
E(t)+\int_{0}^{t}\left(\left\|\nabla u_{\tau}(\tau)\right\|^{2}+\left\|\nabla v_{\tau}(\tau)\right\|^{2}\right. \\
\left.+\|u(\tau)\|_{q}^{q}+\|v(\tau)\|_{q}^{q}\right) d \tau=E(0),
\end{gathered}
$$

where

$$
\begin{aligned}
E(t)= & \frac{1}{2}\left(\left\|u_{t}\right\|^{2}+\left\|v_{t}\right\|^{2}\right)+\frac{1}{p}\left(\|\nabla u\|_{p}^{p}+\|\nabla v\|_{p}^{p}\right) \\
& -\frac{1}{r+2}\|u v\|_{r+2}^{r+2} .
\end{aligned}
$$

Proof. Let $\left\{\omega_{i}\right\}_{i=1}^{\infty}$ be a basis for $W_{0}^{1, p}(\Omega)$. Supposed that $V_{k}$ is the subspace of $W_{0}^{1, p}(\Omega)$ generated by $\left\{\omega_{1}, \omega_{2}, \ldots, \omega_{k}\right\}, k \in N$. We are going to look for the approximate solution

$$
u_{k}(t)=\sum_{i=1}^{k} g_{i k}(t) \omega_{i}, \quad v_{k}(t)=\sum_{i=1}^{k} h_{i k}(t) \omega_{i}
$$


which satisfies the following Cauchy problem:

$$
\begin{gathered}
\int_{\Omega}\left(u_{k}^{\prime \prime}-\operatorname{div}\left(\left|\nabla u_{k}\right|^{p-2} \nabla u_{k}\right)+\left|u_{k}^{\prime}\right|^{q-2} u_{k}^{\prime}-\Delta u_{k}^{\prime}\right) \omega_{i} d x \\
=\int_{\Omega}\left|v_{k}\right|^{r+2}\left|u_{k}\right|^{r} u_{k} \omega_{i} d x, \\
\int_{\Omega}\left(v_{k}^{\prime \prime}-\operatorname{div}\left(\left|\nabla v_{k}\right|^{p-2} \nabla v_{k}\right)+\left|v_{k}^{\prime}\right|^{q-2} v_{k}^{\prime}-\Delta v_{k}^{\prime}\right) \omega_{i} d x \\
=\int_{\Omega}\left|u_{k}\right|^{r+2}\left|v_{k}\right|^{r} v_{k} \omega_{i} d x, \\
u_{k}(0)=u_{0 k}=\sum_{i=1}^{k}\left(u_{0}, \omega_{i}\right) \omega_{i} \longrightarrow u_{0}, \quad \text { in } W_{0}^{1, p}(\Omega), \\
v_{k}(0)=v_{0 k}=\sum_{i=1}^{k}\left(v_{0}, \omega_{i}\right) \omega_{i} \longrightarrow v_{0} \quad \text { in } W_{0}^{1, p}(\Omega), \\
v_{k}^{\prime}(0)=v_{1 k}=\sum_{i=1}^{k}\left(v_{1}, \omega_{i}\right) \omega_{i} \longrightarrow v_{1} \quad \text { in } L^{2}(\Omega), \\
u_{k}^{\prime}(0)=u_{1 k}=\sum_{i=1}^{k}\left(u_{1}, \omega_{i}\right) \omega_{i} \longrightarrow u_{1} \quad \text { in } L^{2}(\Omega), \\
\quad k \longrightarrow \infty, \infty
\end{gathered}
$$

Note that, we can solve the problem (14)-(19) by a Picard's iteration method in ordinary differential equations. Hence, there exists a solution in $\left[0, T_{k}\right)$ for some $T_{k}>0$, and we can extend this solution to the whole interval $[0, T]$ for any given $T>0$ by making use of the a priori estimates below.

Multiplying (14) by $g_{i k}^{\prime}(t)$ and (15) by $h_{i k}^{\prime}(t)$ and summing over $i$ from 1 to $k$, we obtain

$$
\begin{aligned}
& \frac{1}{2} \frac{d}{d t}\left(\left\|u_{k}^{\prime}(t)\right\|^{2}+\left\|\nabla u_{k}\right\|_{p}^{p}\right)+\left\|u_{k}^{\prime}(t)\right\|_{q}^{q}+\left\|\nabla u_{k}^{\prime}(t)\right\|^{2} \\
& \quad=\int_{\Omega}\left|v_{k}\right|^{r+2}\left|u_{k}\right|^{r} u_{k} u_{k}^{\prime} d x, \\
& \frac{1}{2} \frac{d}{d t}\left(\left\|v_{k}^{\prime}(t)\right\|^{2}+\left\|\nabla v_{k}\right\|_{p}^{p}\right)+\left\|v_{k}^{\prime}(t)\right\|_{q}^{q}+\left\|\nabla v_{k}^{\prime}(t)\right\|^{2} \\
& \quad=\int_{\Omega}\left|u_{k}\right|^{r+2}\left|v_{k}\right|^{r} v_{k} v_{k}^{\prime} d x .
\end{aligned}
$$

By summing (20) and (21) and integrating the resulting identity over $[0, t]$, we have

$$
\begin{gathered}
\frac{1}{2}\left(\left\|u_{k}^{\prime}(t)\right\|^{2}+\left\|v_{k}^{\prime}(t)\right\|^{2}+\left\|\nabla u_{k}\right\|_{p}^{p}+\left\|\nabla v_{k}\right\|_{p}^{p}\right) \\
+\int_{0}^{t}\left(\left\|\nabla u_{k}^{\prime}(t)\right\|^{2}+\left\|\nabla v_{k}^{\prime}(t)\right\|^{2}\right. \\
\left.+\left\|u_{k}^{\prime}(\tau)\right\|_{q}^{q}+\left\|v_{k}^{\prime}(\tau)\right\|_{q}^{q}\right) d \tau \\
\leq C_{0}+\int_{0}^{t} \int_{\Omega}\left(\left|v_{k}\right|^{r+2}\left|u_{k}\right|^{r} u_{k} u_{k}^{\prime}\right. \\
\left.+\left|u_{k}\right|^{r+2}\left|v_{k}\right|^{r} v_{k} v_{k}^{\prime}\right) d x d \tau .
\end{gathered}
$$

We estimate the right-hand terms of (22) as follows: we get from Hölder inequality and Lemmas 1 and 2 that

$$
\begin{aligned}
& \left|\int_{0}^{t} \int_{\Omega}\left(\left|v_{k}\right|^{r+2}\left|u_{k}\right|^{r} u_{k} u_{k}^{\prime}+\left|u_{k}\right|^{r+2}\left|v_{k}\right|^{r} v_{k} v_{k}^{\prime}\right) d x d \tau\right| \\
& \leq \int_{0}^{t}\left(\left\|u_{k}^{\prime}(\tau)\right\|^{2}+\left\|v_{k}^{\prime}(\tau)\right\|^{2}\right) d \tau \\
& \quad+\int_{0}^{t} \int_{\Omega}\left|u_{k} v_{k}\right|^{2(r+1)}\left(\left|u_{k}\right|^{2}+\left|v_{k}\right|^{2}\right) d x d \tau \\
& \leq \int_{0}^{t}\left(\left\|u_{k}^{\prime}(\tau)\right\|^{2}+\left\|v_{k}^{\prime}(\tau)\right\|^{2}\right) d \tau \\
& \quad+C \int_{0}^{t}\left(\left\|u_{k}\right\|_{2(r+2)}^{2(r+2)}+\left\|v_{k}\right\|_{2(r+2)}^{2(r+2)}\right) d \tau \\
& \leq C \int_{0}^{t}\left(\left\|u_{k}^{\prime}(\tau)\right\|^{2}+\left\|v_{k}^{\prime}(\tau)\right\|^{2}\right. \\
& \left.+\left\|\nabla u_{k}\right\|_{p}^{2(r+2)}+\left\|\nabla v_{k}\right\|_{p}^{2(r+2)}\right) d \tau \\
& \leq C \int_{0}^{t}\left(\left\|u_{k}^{\prime}(\tau)\right\|^{2}+\left\|v_{k}^{\prime}(\tau)\right\|^{2}\right. \\
& \left.+\left\|\nabla u_{k}\right\|_{p}^{p}+\left\|\nabla v_{k}\right\|_{p}^{p}\right)^{2(r+2) / p} d \tau .
\end{aligned}
$$

It follows from (22) and (23) that

$$
\begin{gathered}
\left\|u_{k}^{\prime}(t)\right\|^{2}+\left\|v_{k}^{\prime}(t)\right\|^{2}+\left\|\nabla u_{k}\right\|_{p}^{p}+\left\|\nabla v_{k}\right\|_{p}^{p} \\
+2 \int_{0}^{t}\left(\left\|u_{k}^{\prime}(\tau)\right\|_{q}^{q}\|+\| v_{k}^{\prime}(\tau) \|_{q}^{q}\right. \\
\left.+\left\|\nabla u_{k}^{\prime}(t)\right\|^{2}+\left\|\nabla v_{k}^{\prime}(t)\right\|^{2}\right) d \tau \\
\leq 2 C_{0}+C \int_{0}^{t}\left(\left\|u_{k}^{\prime}(\tau)\right\|^{2}+\left\|v_{k}^{\prime}(\tau)\right\|^{2}\right. \\
\left.+\left\|\nabla u_{k}\right\|_{p}^{p}+\left\|\nabla v_{k}\right\|_{p}^{p}\right)^{2(r+2) / p} d \tau,
\end{gathered}
$$

which implies that

$$
\begin{gathered}
\left\|u_{k}^{\prime}(t)\right\|^{2}+\left\|v_{k}^{\prime}(t)\right\|^{2}+\left\|\nabla u_{k}\right\|_{p}^{p}+\left\|\nabla v_{k}\right\|_{p}^{p} \\
\leq 2 C_{0}+C \int_{0}^{t}\left(\left\|u_{k}^{\prime}(\tau)\right\|^{2}+\left\|v_{k}^{\prime}(\tau)\right\|^{2}\right. \\
\left.+\left\|\nabla u_{k}\right\|_{p}^{p}+\left\|\nabla v_{k}\right\|_{p}^{p}\right)^{2(r+2) / p} d \tau .
\end{gathered}
$$

We get from (25) and Gronwall type inequality that

$$
\begin{aligned}
\left\|u_{k}^{\prime}(t)\right\|^{2}+\left\|v_{k}^{\prime}(t)\right\|^{2}+\left\|\nabla u_{k}\right\|_{p}^{p}+\left\|\nabla v_{k}\right\|_{p}^{p} \\
\leq\left[2 C_{0}-\frac{2(r+2)-p}{p} C t\right]^{-p /(2(r+2)-p)} .
\end{aligned}
$$


Thus, we deduce from (26) that there exists a time $T>0$ such that

$\left\|u_{k}^{\prime}(t)\right\|^{2}+\left\|v_{k}^{\prime}(t)\right\|^{2}+\left\|\nabla u_{k}\right\|_{p}^{p}+\left\|\nabla v_{k}\right\|_{p}^{p} \leq C_{1}, \quad \forall t \in[0, T]$,

where $C_{1}$ is a positive constant independent of $k$.

We have from (24) and (26) that

$$
\begin{aligned}
2 \int_{0}^{t}( & \left\|u_{k}^{\prime}(\tau)\right\|_{q}^{q}+\left\|v_{k}^{\prime}(\tau)\right\|_{q}^{q} \\
& \left.+\left\|\nabla u_{k}^{\prime}(\tau)\right\|^{2}+\left\|\nabla v_{k}^{\prime}(\tau)\right\|^{2}\right) d \tau \leq C_{2}, \quad \forall t \in[0, T]
\end{aligned}
$$

It follows from (27) and (28) that

$$
\begin{gathered}
\left\|u_{k}^{\prime}(t)\right\|^{2} \leq C_{1}, \quad\left\|v_{k}^{\prime}(t)\right\|^{2} \leq C_{1}, \\
\left\|\nabla u_{k}\right\|_{p}^{p} \leq C_{1}, \quad\left\|\nabla v_{k}\right\|_{p}^{p} \leq C_{1} .
\end{gathered}
$$

$u_{k}^{\prime}(t)$ and $v_{k}^{\prime}(t)$ are bounded in $L^{2}\left([0, T] ; L^{q}(\Omega)\right)$

$$
\text { and } L^{2}\left([0, T] ; H_{0}^{1}(\Omega)\right) \text {. }
$$

Using the same process as the proof of Theorem 2.1 in paper [18], we derive that $[u(t), v(t)]$ is a local solution of the problem (1)-(5). By (20) and (21), we conclude that (11) is valid.

\section{Global Existence}

In order to state our main results, we first introduce the following functionals:

$$
\begin{gathered}
J([u, v])=\frac{1}{p}\left(\|\nabla u\|_{p}^{p}+\|\nabla v\|_{p}^{p}\right)-\frac{1}{r+2}\|u v\|_{r+2}^{r+2}, \\
K([u, v])=\left(\|\nabla u\|_{p}^{p}+\|\nabla v\|_{p}^{p}\right)-2\|u v\|_{r+2}^{r+2}
\end{gathered}
$$

for $[u, v] \in W_{0}^{1, p}(\Omega) \times W_{0}^{1, p}(\Omega)$.

We put that

$$
\begin{gathered}
d=\inf \left\{\sup _{\lambda \geq 0} J[\lambda, v]\right):[u, v] \in W_{0}^{1, p}(\Omega) \\
\left.\times W_{0}^{1, p}(\Omega) /\{[0,0]\}\right\} .
\end{gathered}
$$

Then, we are able to define the stable set as follows for problem (1)-(5):

$$
\begin{gathered}
W=\left\{[u, v] \in W_{0}^{1, p}(\Omega) \times W_{0}^{1, p}(\Omega) \mid K([u, v])>0,\right. \\
J([u, v])<d\} \cup\{[0,0]\} .
\end{gathered}
$$

We denote the total energy related to (1) and (2) by (12), and

$$
\begin{aligned}
E(0)= & \frac{1}{2}\left(\left\|u_{1}\right\|^{2}+\left\|v_{1}\right\|^{2}\right)+\frac{1}{p}\left(\left\|\nabla u_{0}\right\|_{p}^{p}+\left\|\nabla v_{0}\right\|_{p}^{p}\right) \\
& -\frac{1}{r+2}\left\|u_{0} v_{0}\right\|_{r+2}^{r+2}
\end{aligned}
$$

Lemma 5. Let $[u, v]$ be a solution to problem (1)-(5); then, $E(t)$ is a nonincreasing function for $t>0$ and

$$
\frac{d}{d t} E(t)=-\left(\left\|u_{t}\right\|_{q}^{q}+\left\|v_{t}\right\|_{q}^{q}+\left\|\nabla u_{t}\right\|_{2}^{2}+\left\|\nabla v_{t}\right\|_{2}^{2}\right)
$$

We have from (11) that $E(t)$ is the primitive of an integrable function. Therefore, $E(t)$ is absolutely continuous, and equality (35) is satisfied.

Lemma 6. Supposed that $[u, v] \in W_{0}^{1, p}(\Omega) \times W_{0}^{1, p}(\Omega)$, and $p<2(r+2) \leq n p /(n-p)$ if $n \geq p ; p<2(r+2)<+\infty$ if $n<p$, then $d>0$.

Proof. Since

$$
J(\lambda[u, v])=\frac{\lambda^{p}}{p}\left(\|\nabla u\|_{p}^{p}+\|\nabla v\|_{p}^{p}\right)-\frac{\lambda^{2(r+2)}}{r+2}\|u v\|_{r+2}^{r+2},
$$

so we get

$$
\frac{d}{d \lambda} J(\lambda[u, v])=\lambda^{p-1}\left(\|\nabla u\|_{p}^{p}+\|\nabla v\|_{p}^{p}\right)-2 \lambda^{2 r+3}\|u v\|_{r+2}^{r+2} .
$$

In case $u v \neq 0$, let $(d / d \lambda) J(\lambda[u, v])=0$, which implies that

$$
\lambda_{1}=\left(\frac{\|\nabla u\|_{p}^{p}+\|\nabla v\|_{p}^{p}}{2\|u v\|_{r+2}^{r+2}}\right)^{1 /(2 r-p+4)} .
$$

As $\lambda=\lambda_{1}$, an elementary calculation shows that $\left.\left(d^{2} / d \lambda^{2}\right) J(\lambda[u, v])\right|_{\lambda=\lambda_{1}}<0$. Therefore, we have that

$$
\begin{aligned}
\left.\sup _{\lambda \geq 0} J[u, v]\right) & \left(\lambda\left[\lambda_{1}[u, v]\right)\right. \\
& =J(r+2)-p \\
& \left.=\frac{2\left(r+\nabla u\left\|_{p}^{p}+\right\| \nabla v \|_{p}^{p}\right.}{2 p(r+2)}\right)^{(2 r+4) /(2 r-p+4)}
\end{aligned}
$$

It follows from Hölder inequality and Lemma 1 that

$$
\begin{aligned}
\|u v\|_{r+2}^{p / 2} & \leq\|u\|_{2(r+2)}^{p / 2}\|v\|_{2(r+2)}^{p / 2} \\
& \leq \frac{1}{2}\left(\|u\|_{2(r+2)}^{p}+\|v\|_{2(r+2)}^{p}\right) \\
& \leq C\left(\|\nabla u\|_{p}^{p}+\|\nabla v\|_{p}^{p}\right) .
\end{aligned}
$$

We get from (39) and (40) that

$$
\sup _{\lambda \geq 0} J[\lambda[u, v]) \geq \frac{2(r+2)-p}{2 p(r+2)}\left(2^{p /(2 r+4)} C\right)^{-(2 r+4) /(2 r-p+4)}>0 .
$$

In case $u v=0$ and $u=0$ or $v=0$, then

$$
J(\lambda[u, v])=\frac{\lambda^{p}}{p}\left(\|\nabla u\|_{p}^{p}+\|\nabla v\|_{p}^{p}\right) .
$$

is the total energy of the initial data. 
Therefore, we have

$$
J(\lambda[u, v])=+\infty
$$

We conclude from (41) and (43) that

$$
d \geq \frac{2(r+2)-p}{2 p(r+2)}\left(2^{p /(2 r+4)} C\right)^{-(2 r+4) /(2 r-p+4)}>0 .
$$

Thus, we complete the proof of Lemma 6.

Lemma 7. Supposed that $p<2(r+2) \leq n p /(n-p)$ for $n \geq p$ and $p<2(r+2)<+\infty$ for $n<p$, if $\left[u_{0}, v_{0}\right] \in W$, $\left[u_{1}, v_{1}\right] \in$ $L^{2}(\Omega) \times L^{2}(\Omega)$ and $E(0)<d$, then $[u, v] \in W$ for $\forall t \in[0, T)$.

Proof. Assume that there exists a number $t^{*} \in(0, T)$ such that $[u(t), v(t)] \in W$ on $\left[0, t^{*}\right)$ and $u\left(t^{*}\right) \notin W$. Then, in virtue of the continuity of $u(t)$, we see $u\left(t^{*}\right) \in \partial W$, where $\partial W$ denotes the boundary of domain $W$. From the definition of $W$ and the continuity of $J([u(t), v(t)])$ and $K([u(t), v(t)])$ in $t$, we have either

$$
J\left(\left[u\left(t^{*}\right), v\left(t^{*}\right)\right]\right)=d
$$

or

$$
K\left(\left[u\left(t^{*}\right), v\left(t^{*}\right)\right]\right)=0 .
$$

It follows from (12) and (30) that

$$
J\left(\left[u\left(t^{*}\right), v\left(t^{*}\right)\right]\right) \leq E\left(t^{*}\right) \leq E(0)<d .
$$

So, case (45) is impossible.

Assume that (46) holds; then, we get that

$$
\begin{aligned}
& \frac{d}{d \lambda} J\left(\lambda\left[u\left(t^{*}\right), v\left(t^{*}\right)\right]\right) \\
& \quad=\lambda^{p-1}\left(1-\lambda^{2 r-p+4}\right)\left(\|\nabla u\|_{p}^{p}+\|\nabla v\|_{p}^{p}\right) .
\end{aligned}
$$

We obtain from $(d / d \lambda) J\left(\lambda\left[u\left(t^{*}\right), v\left(t^{*}\right)\right]\right)=0$ that $\lambda=1$. Since

$$
\begin{aligned}
& \left.\frac{d^{2}}{d \lambda^{2}} J\left(\lambda\left[u\left(t^{*}\right), v\left(t^{*}\right)\right]\right)\right|_{\lambda=1} \\
& \quad=-[(2(r+2)-p)+(2 r+3)]<0 .
\end{aligned}
$$

Consequently, we get from (47) that

$$
\sup _{\lambda \geq 0} J\left(\lambda\left[u\left(t^{*}\right), v\left(t^{*}\right)\right]\right)=J\left(\left[u\left(t^{*}\right), v\left(t^{*}\right)\right]\right)<d,
$$

which contradicts the definition of $d$. Hence, case (46) is impossible as well. Thus we conclude that $[u(t), v(t)] \in W$ on $[0, T)$.

Theorem 8 (global solution). Supposed that $p<2(r+2) \leq$ $n p /(n-p)$ as $n \geq p$ and $p<2(r+2)<+\infty$ as $n<p$, and $[u(t), v(t)]$ is a local solution of problem (1)-(5) on $[0, T)$. If $\left[u_{0}, v_{0}\right] \in W,\left[u_{1}, v_{1}\right] \in L^{2}(\Omega) \times L^{2}(\Omega)$ and $E(0)<d$, then $[u(t), v(t)]$ is a global solution of problem (1)-(5).
Proof. It suffices to show that $\left\|u_{t}\right\|^{2}+\left\|v_{t}\right\|^{2}+\|\nabla u\|_{p}^{p}+\|\nabla v\|_{p}^{p}$ is bounded uniformly with respect to $t$. Under the hypotheses in Theorem 8 , we get from Lemma 7 that $[u, v] \in W$ on $[0, T)$. So the following formula holds on $[0, T)$ :

$$
\begin{aligned}
J([u, v]) & =\frac{1}{p}\left(\|\nabla u\|_{p}^{p}+\|\nabla v\|_{p}^{p}\right)-\frac{1}{r+2}\|u v\|_{r+2}^{r+2} \\
& \geq \frac{2(r+2)-p}{2 p(r+2)}\left(\|\nabla u\|_{p}^{p}+\|\nabla v\|_{p}^{p}\right) .
\end{aligned}
$$

We have from (51) that

$$
\begin{aligned}
& \frac{1}{2}\left(\left\|u_{t}\right\|^{2}+\left\|v_{t}\right\|^{2}\right)+\frac{2(r+2)-p}{2 p(r+2)}\left(\|\nabla u\|_{p}^{p}+\|\nabla v\|_{p}^{p}\right) \\
& \quad \leq \frac{1}{2}\left(\left\|u_{t}\right\|^{2}+\left\|v_{t}\right\|^{2}\right)+J([u(t), v(t)]) \\
& \quad=E(t) \leq E(0)<d .
\end{aligned}
$$

Hence, we get

$$
\begin{aligned}
& \left(\left\|u_{t}\right\|^{2}+\left\|v_{t}\right\|^{2}\right)+\left(\|\nabla u\|_{p}^{p}+\|\nabla v\|_{p}^{p}\right) \\
& \quad \leq \max \left(2, \frac{2 p(r+2)}{2(r+2)-p}\right) d<+\infty .
\end{aligned}
$$

The above inequality and the continuation principle lead to the global existence of the solution $[u, v]$ for problem (1)-(5).

\section{Asymptotic Behavior of Global Solutions}

The following lemma plays an important role in studying the decay estimate of global solutions for the problem (1)-(5).

Lemma 9 (see [9]). Suppose that $\varphi(t)$ is a nonincreasing nonnegative function on $[0,+\infty)$ and satisfies

$$
\varphi(t)^{r+1} \leq k(\varphi(t)-\varphi(t+1)), \quad \forall t \geq 0 .
$$

Then, $\varphi(t)$ has the decay property

$$
\varphi(t) \leq\left[\frac{r}{k}(t-1)+M^{-r}\right]^{-1 / r}, \quad \forall t \geq 1,
$$

where $k, r>0$ are constants and $M=\max _{t \in[0,1]} \varphi(t)$.

Lemma 10. Under the assumptions of Theorem 8, if initial value $\left[u_{0}, v_{0}\right] \in W$ and $\left[u_{1}, v_{1}\right] \in L^{2}(\Omega) \times L^{2}(\Omega)$ are sufficiently small such that

$$
C^{2(r+2)}\left(\frac{2 p(r+2)}{2 p(r+2)-p} E(0)\right)^{(2(r+2)-p) / p}<1,
$$

then

$$
\left(\|\nabla u\|_{p}^{p}+\|\nabla v\|_{p}^{p}\right) \leq \frac{1}{\theta} K([u, v])
$$

where $\theta=1-C^{2(r+2)}((2 p(r+2) /(2 p(r+2)-$ $p)) E(0))^{(2(r+2)-p) / p}>0$ is a positive constant and $C$ is the optimal Sobolev's constant from $W_{0}^{1, p}(\Omega)$ to $L^{2(r+2)}(\Omega)$. 
Proof. We have from Lemma 1 and (52) that

$$
\begin{aligned}
2\|u v\|_{r+2}^{r+2} \leq & 2\|u\|_{2(r+2)}^{r+2}\|v\|_{2(r+2)}^{r+2} \\
\leq & \|u\|_{2(r+2)}^{2(r+2)}+\|v\|_{2(r+2)}^{2(r+2)} \\
\leq & C^{2(r+2)}\left(\|\nabla u\|_{p}^{2(r+2)}+\|\nabla v\|_{p}^{2(r+2)}\right) \\
\leq & C^{2(r+2)}\left(\|\nabla u\|_{p}^{2(r+2)-p}\|\nabla u\|_{p}^{p}\right. \\
& \left.+\|\nabla v\|_{p}^{2(r+2)-p}\|\nabla v\|_{p}^{p}\right) \\
\leq & C^{2(r+2)}\left(\frac{2 p(r+2)}{2 p(r+2)-p} E(0)\right)^{(2(r+2)-p) / p} \\
& \times\left(\|\nabla u\|_{p}^{p}+\|\nabla v\|_{p}^{p}\right) .
\end{aligned}
$$

Therefore, we get from (58) and (31) that

$$
\begin{gathered}
{\left[1-C^{2(r+2)}\left(\frac{2 p(r+2)}{2 p(r+2)-p} E(0)\right)^{(2(r+2)-p) / p}\right]} \\
\times\left(\|\nabla u\|_{p}^{p}+\|\nabla v\|_{p}^{p}\right) \leq K([u, v]) .
\end{gathered}
$$

Let

$$
\theta=1-C^{2(r+2)}\left(\frac{2 p(r+2)}{2 p(r+2)-p} E(0)\right)^{(2(r+2)-p) / p}>0
$$

then, we have from (59) that

$$
\|\nabla u\|_{p}^{p}+\|\nabla v\|_{p}^{p} \leq \frac{1}{\theta} K([u, v]) .
$$

Theorem 11. Under the assumptions of Theorem 8, if $p<q<$ $r+2$ and (56) hold, then the global solution $[u, v]$ in $W$ of the problem (1)-(5) has the following decay property:

$$
E(t) \leq\left[\frac{p-2}{p C}(t-1)+M^{(p+q-p q) / p}\right]^{p /(p+q-p q)}, \quad \forall t>1,
$$

where $M=\max _{t \in[0,1]} E(t)>0$ is some constant depending only on $\left[u_{0}, v_{0}\right]$ and $\left[u_{1}, v_{1}\right]$.

Proof. Multiplying (1) by $u_{t}$ and (2) by $v_{t}$ and integrating over $\Omega \times[t, t+1]$, and summing up together, we get

$$
\begin{aligned}
\int_{t}^{t+1}\left(\left\|u_{t}(s)\right\|_{q}^{q}+\left\|v_{t}(s)\right\|_{q}^{q}+\left\|\nabla u_{t}(s)\right\|_{2}^{2}\right. \\
\left.+\left\|\nabla v_{t}(s)\right\|_{2}^{2}\right) d s=E(t)-E(t+1) .
\end{aligned}
$$

Thus, there exists $t_{1} \in[t, t+1 / 4], t_{2} \in[t+3 / 4, t+1]$ such that

$$
\begin{gathered}
4\left(\left\|u_{t}\left(t_{i}\right)\right\|_{q}^{q}+\left\|v_{t}\left(t_{i}\right)\right\|_{q}^{q}+\left\|\nabla u_{t}\left(t_{i}\right)\right\|_{2}^{2}+\left\|\nabla v_{t}\left(t_{i}\right)\right\|_{2}^{2}\right) \\
=E(t)-E(t+1), \quad i=1,2 .
\end{gathered}
$$

On the other hand, we multiply (1) by $u$ and (2) by $v$ and integrate over $\Omega \times\left[t_{1}, t_{2}\right]$. Adding them together, we obtain

$$
\begin{aligned}
\int_{t_{1}}^{t_{2}} K([u, v]) d s= & \int_{t_{1}}^{t_{2}}\left\|u_{t}\right\|^{2} d s+\int_{t_{1}}^{t_{2}}\left\|v_{t}\right\|^{2} d s \\
& +\left(u_{t}\left(t_{1}\right), u\left(t_{1}\right)\right)-\left(u_{t}\left(t_{2}\right), u\left(t_{2}\right)\right) \\
& +\left(v_{t}\left(t_{1}\right), v\left(t_{2}\right)\right)-\left(v_{t}\left(t_{2}\right) v\left(t_{2}\right)\right) \\
& -\left(\int_{t_{1}}^{t_{2}} \int_{\Omega}\left|u_{t}\right|^{q-2} u_{t} u d x d s\right. \\
& \left.+\int_{t_{1}}^{t_{2}} \int_{\Omega}\left|v_{t}\right|^{q-2} v_{t} v d x d s\right) \\
& -\int_{t_{1}}^{t_{2}} \int_{\Omega} \nabla u_{t} \nabla u d x d s-\int_{t_{1}}^{t_{2}} \int_{\Omega} \nabla v_{t} \nabla v d x d s .
\end{aligned}
$$

From (63), Sobolev inequality, and Hölder inequality, we have

$$
\begin{aligned}
& \int_{t_{1}}^{t_{2}}\left\|u_{t}\right\|^{2} d s \leq C \int_{t_{1}}^{t_{2}}\left\|\nabla u_{t}\right\|^{2} d s \leq C(E(t)-E(t+1)), \\
& \int_{t_{1}}^{t_{2}}\left\|v_{t}\right\|^{2} d s \leq C \int_{t_{1}}^{t_{2}}\left\|\nabla v_{t}\right\|^{2} d s \leq C(E(t)-E(t+1)) .
\end{aligned}
$$

We get from (52), (64), and Lemmas 1 and 2 that

$$
\begin{aligned}
\left|u_{t}\left(t_{i}\right), u\left(t_{i}\right)\right| \leq & \left\|u_{t}\left(t_{i}\right)\right\| \cdot\left\|u\left(t_{i}\right)\right\| \leq C\left\|\nabla u_{t}\left(t_{i}\right)\right\| \cdot\left\|\nabla u\left(t_{i}\right)\right\|_{p} \\
\leq & C(E(t)-E(t+1))^{1 / 2} \sup _{t \leq s \leq t+1} E(s)^{1 / p} \\
\leq & C(\varepsilon)(E(t)-E(t+1))^{p / 2(p-1)} \\
& +\varepsilon \sup _{t \leq s \leq t+1} E(s), \quad i=1,2, \\
\left|\left(v_{t}\left(t_{i}\right), v\left(t_{i}\right)\right)\right| \leq & \left\|v_{t}\left(t_{i}\right)\right\| \cdot\left\|v\left(t_{i}\right)\right\| \leq C\left\|\nabla v_{t}\left(t_{i}\right)\right\| \cdot\left\|\nabla v\left(t_{i}\right)\right\|_{p} \\
\leq & C(E(t)-E(t+1))^{1 / 2} \sup _{t \leq s \leq t+1} E(s)^{1 / p} \\
\leq & C(\varepsilon)(E(t)-E(t+1))^{p / 2(p-1)} \\
& +\varepsilon \sup _{t \leq s \leq t+1} E(s), \quad i=1,2 .
\end{aligned}
$$

From Hölder inequality and Lemma 2,we get

$$
\begin{aligned}
\left.\left|\int_{t_{1}}^{t_{2}} \int_{\Omega}\right| u_{t}\right|^{q-2} u_{t} u d x d s \mid & \leq \int_{t_{1}}^{t_{2}}\left\|u_{t}\right\|_{q}^{q-1}\|u\|_{q} d s \\
& \leq\left(\int_{t_{1}}^{t_{2}}\left\|u_{t}\right\|_{q}^{q} d s\right)^{(q-1) / q}\left(\int_{t_{1}}^{t_{2}}\|u\|_{q}^{q} d s\right)^{1 / q} \\
& \leq C(\varepsilon) \int_{t_{1}}^{t_{2}}\left\|u_{t}\right\|_{q}^{q} d s+\varepsilon \int_{t_{1}}^{t_{2}}\|u\|_{q}^{q} d s,
\end{aligned}
$$




$$
\begin{aligned}
\left.\left|\int_{t_{1}}^{t_{2}} \int_{\Omega}\right| v_{t}\right|^{q-2} v_{t} v d x d s \mid & \leq \int_{t_{1}}^{t_{2}}\left\|v_{t}\right\|_{q}^{q-1}\|v\|_{q} d s \\
& \leq\left(\int_{t_{1}}^{t_{2}}\left\|v_{t}\right\|_{q}^{q} d s\right)^{(q-1) / q}\left(\int_{t_{1}}^{t_{2}}\|v\|_{q}^{q} d s\right)^{1 / q} \\
& \leq C(\varepsilon) \int_{t_{1}}^{t_{2}}\left\|v_{t}\right\|_{q}^{q} d s+\varepsilon \int_{t_{1}}^{t_{2}}\|v\|_{q}^{q} d s .
\end{aligned}
$$

Since $p<q<r+2$ and the property of the function $f(x)=\alpha^{x} / x, \alpha \geq 0, x>0$, we obtain

$$
\frac{\|u\|_{q}^{q}}{q} \leq C \frac{\|u\|_{p}^{p}}{p}+C \frac{\|u\|_{r+2}^{r+2}}{r+2}, \quad \frac{\|v\|_{q}^{q}}{q} \leq C \frac{\|v\|_{p}^{p}}{p}+C \frac{\|v\|_{r+2}^{r+2}}{r+2} .
$$

We conclude from (69), (70), $[u, v] \in W$, and Lemma 1 that

$$
\begin{aligned}
\|u\|_{q}^{q}+\|v\|_{q}^{q} & \leq C\left(\|u\|_{p}^{p}+\|u\|_{r+2}^{r+2}+\|v\|_{p}^{p}+\|v\|_{r+2}^{r+2}\right) \\
& \leq C\left(\|u\|_{p}^{p}+\|\nabla u\|_{p}^{p}+\|v\|_{p}^{p}+\|\nabla v\|_{p}^{p}\right) \\
& \leq C\left(\|\nabla u\|_{p}^{p}+\|\nabla v\|_{p}^{p}\right) \leq C E(t) .
\end{aligned}
$$

It follows from (63), (68), (69), and (71) that

$$
\begin{aligned}
& \left|-\left(\int_{t_{1}}^{t_{2}} \int_{\Omega}\left|u_{t}\right|^{q-2} u_{t} u d x d s+\int_{t_{1}}^{t_{2}} \int_{\Omega}\left|v_{t}\right|^{q-2} v_{t} v d x d s\right)\right| \\
& \leq C(\varepsilon)(E(t)-E(t+1))+\varepsilon C \int_{t_{1}}^{t_{2}} E(s) d s,
\end{aligned}
$$

and we obtain from (63), Sobolev inequality, Hölder inequality, and Lemma 2 that

$$
\begin{aligned}
\left|-\int_{t_{1}}^{t_{2}} \int_{\Omega} \nabla u_{t} \nabla u d s\right| \leq & \int_{t_{1}}^{t_{2}}\left\|\nabla u_{t}\right\| \cdot\|\nabla u\| d s \\
\leq & \left(\int_{t_{1}}^{t_{2}}\left\|\nabla u_{t}\right\|^{2} d s\right)^{1 / 2}\left(\int_{t_{1}}^{t_{2}}\|\nabla u\|^{2} d s\right)^{1 / 2} \\
& \leq C(E(t)-E(t+1))^{1 / 2}\left(\int_{t_{1}}^{t_{2}}\|\nabla u\|_{p}^{2} d s\right)^{1 / 2} \\
& \leq C(E(t)-E(t+1))^{1 / 2}\left(\int_{t_{1}}^{t_{2}}\|\nabla u\|_{p}^{p} d s\right)^{1 / p} \\
& \leq C(E(t)-E(t+1))^{p / 2(p-1)} \\
& +\varepsilon \int_{t_{1}}^{t_{2}}\|\nabla u\|_{p}^{p} d s .
\end{aligned}
$$

Similarly, we have the following formula:

$\left|-\int_{t_{1}}^{t_{2}} \int_{\Omega} \nabla v_{t} \nabla v d s\right| \leq \int_{t_{1}}^{t_{2}}\left\|\nabla v_{t}\right\| \cdot\|\nabla v\| d s$

$$
\leq\left(\int_{t_{1}}^{t_{2}}\left\|\nabla v_{t}\right\|^{2} d s\right)^{1 / 2}\left(\int_{t_{1}}^{t_{2}}\|\nabla v\|^{2} d s\right)^{1 / 2}
$$

$$
\begin{aligned}
\leq & C(E(t)-E(t+1))^{1 / 2}\left(\int_{t_{1}}^{t_{2}}\|\nabla v\|_{p}^{2} d s\right)^{1 / 2} \\
\leq & C(E(t)-E(t+1))^{1 / 2}\left(\int_{t_{1}}^{t_{2}}\|\nabla v\|_{p}^{p} d s\right)^{1 / p} \\
\leq & C(E(t)-E(t+1))^{p / 2(p-1)} \\
& +\varepsilon \int_{t_{1}}^{t_{2}}\|\nabla v\|_{p}^{p} d s .
\end{aligned}
$$

We get from (57), (73), and (74) that

$$
\begin{aligned}
& \left|\int_{t_{1}}^{t_{2}} \int_{\Omega}\left(\nabla u_{t} \nabla u+\nabla v_{t} \nabla v\right) d s\right| \\
& \leq C(E(t)-E(t+1))^{p / 2(p-1)}+\varepsilon \int_{t_{1}}^{t_{2}}\left(\|\nabla u\|_{p}^{p}+\|\nabla v\|_{p}^{p}\right) d s \\
& \leq C(E(t)-E(t+1))^{p / 2(p-1)}+\frac{\varepsilon}{\theta} \int_{t_{1}}^{t_{2}} K([u, v]) d s .
\end{aligned}
$$

Choosing small enough $\varepsilon$, we have from (65), (66), (67), (72), and (75) that

$$
\begin{aligned}
\int_{t_{1}}^{t_{2}} K([u, v]) d s \leq & C[(E(t)-E(t+1)) \\
& \left.+(E(t)-E(t+1))^{p / 2(p-1)}\right] \\
& +\varepsilon \sup _{t \leq s \leq t+1} E(s)+\varepsilon \int_{t_{1}}^{t_{2}} E(s) d s .
\end{aligned}
$$

It follows from (30) and (31) that

$$
\begin{aligned}
J([u, v])= & \frac{2(r+2)-p}{2 p(r+2)}\left(\|\nabla u\|_{p}^{p}+\|\nabla v\|_{p}^{p}\right) \\
& +\frac{1}{2(r+2)} K([u, v]) .
\end{aligned}
$$

On the other hand, from (12) and using (57) and (77), we deduce that

$$
\begin{aligned}
E(t)= & \frac{1}{2}\left(\left\|u_{t}\right\|^{2}+\left\|v_{t}\right\|^{2}\right)+J([u, v]) \\
= & \frac{1}{2}\left(\left\|u_{t}\right\|^{2}+\left\|v_{t}\right\|^{2}\right)+\frac{2(r+2)-p}{2 p(r+2)} \\
& \times\left(\|\nabla u\|_{p}^{p}+\|\nabla v\|_{p}^{p}\right)+\frac{1}{2(r+2)} K([u, v]) \\
\leq & \frac{1}{2}\left(\left\|u_{t}\right\|^{2}+\left\|v_{t}\right\|^{2}\right)+\left(\frac{2(r+2)-p}{2 \theta p(r+2)}+\frac{1}{2(r+2)}\right) \\
& \times K([u, v]) .
\end{aligned}
$$


By integrating (78) over $\left[t_{1}, t_{2}\right]$, we obtain

$$
\begin{aligned}
\int_{t_{1}}^{t_{2}} E(s) d s \leq & \frac{1}{2} \int_{t_{1}}^{t_{2}}\left(\left\|u_{t}\right\|^{2}+\left\|v_{t}\right\|^{2}\right) d s \\
& +\left(\frac{2(r+2)-p}{2 \theta p(r+2)}+\frac{1}{2(r+2)}\right) \int_{t_{1}}^{t_{2}} K([u, v]) d s .
\end{aligned}
$$

For small enough $\varepsilon$, we have from (76) and (79) that

$$
\begin{aligned}
\int_{t_{1}}^{t_{2}} E(s) d s \\
\leq C\left[(E(t)-E(t+1))+(E(t)-E(t+1))^{p /(2(p-1))}\right] \\
\quad+\varepsilon \sup _{t \leq s \leq t+1} E(s) .
\end{aligned}
$$

Thus, there exists $t^{*} \in\left[t_{1}, t_{2}\right]$, such that

$$
\begin{aligned}
E\left(t^{*}\right) \leq & C\left[(E(t)-E(t+1))+(E(t)-E(t+1))^{p / 2(p-1)}\right] \\
& +\varepsilon \sup _{t \leq s \leq t+1} E(s)
\end{aligned}
$$

Multiplying (1) by $u_{t}$ and (2) by $v_{t}$ and integrating over $\Omega \times$ $\left[t^{*}, t_{2}\right]$, and summing up, we get

$$
E\left(t_{2}\right)=E\left(t^{*}\right)-\int_{t^{*}}^{t_{2}}\left(\left\|u_{t}\right\|_{q}^{q}+\left\|v_{t}\right\|_{q}^{q}+\left\|\nabla u_{t}\right\|^{2}+\left\|\nabla v_{t}\right\|^{2}\right) d s .
$$

Therefore, we obtain from (63), (81), and (82) that

$$
\begin{aligned}
\sup _{t \leq s \leq t+1} E(s) \leq C[ & (E(t)-E(t+1)) \\
& \left.+(E(t)-E(t+1))^{p / 2(p-1)}\right]+\varepsilon \sup _{t \leq s \leq t+1} E(s) .
\end{aligned}
$$

Choosing small enough $\varepsilon$, we have from (83) that

$$
\begin{aligned}
\sup _{t \leq s \leq t+1} E(s) \leq C[ & (E(t)-E(t+1)) \\
& \left.+(E(t)-E(t+1))^{p / 2(p-1)}\right] .
\end{aligned}
$$

Since $p>2$ and $E(t)<E(0)$, we get

$$
\sup _{t \leq s \leq t+1} E(s) \leq C(E(t)-E(t+1))^{p / 2(p-1)} .
$$

Consequently,

$$
\sup _{t \leq s \leq t+1} E(s)^{(2(p-1)) / p} \leq C(E(t)-E(t+1)) .
$$

Thus, applying Lemma 9 to (86), we get

$$
E(t) \leq\left[\frac{p-2}{p C}(t-1)+M^{(p-2) / p}\right]^{p /(2-p)}, \quad \forall t>1,
$$

where $M=\max _{t \in[0,1]} E(t)>0$ is some constant depending only on $\left[u_{0}, v_{0}\right]$ and $\left[u_{1}, v_{1}\right]$.

\section{Acknowledgments}

This research was supported by the National Natural Science Foundation of China (no. 61273016), The Natural Science Foundation of Zhejiang Province (no. Y6100016), The Middle-aged and Young Leader in Zhejiang University of Science and Technology (2008-2012), and the Interdisciplinary Pre-research Project of Zhejiang University of Science and Technology (2010-2012).

\section{References}

[1] L. A. Medeiros and M. M. Miranda, "Weak solutions for a system of nonlinear Klein-Gordon equations," Annali di Matematica Pura ed Applicata, vol. 146, pp. 173-183, 1987.

[2] M. M. Cavalcanti, V. N. D. Cavalcanti, J. S. P. Filho, and J. A. Soriano, "Existence and uniform decay of solutions of a parabolichyperbolic equation with nonlinear boundary damping and boundary source term," Communications in Analysis and Geometry, vol. 10, no. 3, pp. 451-466, 2002.

[3] M. M. Cavalcanti, V. N. Domingos Cavalcanti, and P. Martinez, "Existence and decay rate estimates for the wave equation with nonlinear boundary damping and source term," Journal of Differential Equations, vol. 203, no. 1, pp. 119-158, 2004.

[4] M. M. Cavalcanti and V. N. Domingos Cavalcanti, "Existence and asymptotic stability for evolution problems on manifolds with damping and source terms," Journal of Mathematical Analysis and Applications, vol. 291, no. 1, pp. 109-127, 2004.

[5] K. Agre and M. A. Rammaha, "Systems of nonlinear wave equations with damping and source terms," Differential and Integral Equations, vol. 19, no. 11, pp. 1235-1270, 2006.

[6] C. O. Alves, M. M. Cavalcanti, V. N. Domingos Cavalcanti, M. A. Rammaha, and D. Toundykov, "On existence, uniform decay rates and blow up for solutions of systems of nonlinear wave equations with damping and source terms," Discrete and Continuous Dynamical Systems, vol. 2, no. 3, pp. 583-608, 2009.

[7] M. A. Rammaha and Z. Wilstein, "Hadamard well-posedness for wave equations with $p$-Laplacian damping and supercritical sources," Advances in Differential Equations, vol. 17, no. 1-2, pp. 105-150, 2012.

[8] Z. Yang, "Existence and asymptotic behaviour of solutions for a class of quasi-linear evolution equations with non-linear damping and source terms," Mathematical Methods in the Applied Sciences, vol. 25, no. 10, pp. 795-814, 2002.

[9] K. Ono, "Blow up phenomenon for nonlinear dissipative wave equatins," Journal of Mathematics, Tokushima University, vol. 30, pp. 19-43, 1996.

[10] F. Dell'Oro and V. Pata, "Long-term analysis of strongly damped nonlinear wave equations," Nonlinearity, vol. 24 , no. 12 , pp. 3413-3435, 2011.

[11] T. F. Ma and J. A. Soriano, "On weak solutions for an evolution equation with exponential nonlinearities," Nonlinear Analysis, vol. 37, no. 8, Ser. A: Theory Methods, pp. 1029-1038, 1999.

[12] M. A. Rammaha, "The influence of damping and source terms on solutions of nonlinear wave equations," Boletim da Sociedade Paranaense de Matemática, vol. 25, no. 1-2, pp. 77-90, 2007.

[13] C. O. Alves and M. M. Cavalcanti, "On existence, uniform decay rates and blow up for solutions of the 2-D wave equation with exponential source," Calculus of Variations and Partial Differential Equations, vol. 34, no. 3, pp. 377-411, 2009. 
[14] L. Bociu, M. Rammaha, and D. Toundykov, "On a wave equation with supercritical interior and boundary sources and damping terms," Mathematische Nachrichten, vol. 284, no. 16, pp. 20322064, 2011.

[15] D. H. Sattinger, "On global solution of nonlinear hyperbolic equations," Archive for Rational Mechanics and Analysis, vol. 30, pp. 148-172, 1968.

[16] L. E. Payne and D. H. Sattinger, "Saddle points and instability of nonlinear hyperbolic equations," Israel Journal of Mathematics, vol. 22, no. 3-4, pp. 273-303, 1975.

[17] M. Nakao, "A difference inequality and its application to nonlinear evolution equations," Journal of the Mathematical Society of Japan, vol. 30, no. 4, pp. 747-762, 1978.

[18] E. Piskin and N. Polat, "Global existence, decay and blow-up solutions for coupled nonlinear wave equations with damping and source terms," Turkish Journal of Mathematics, vol. 30, pp. $1-19,2013$. 


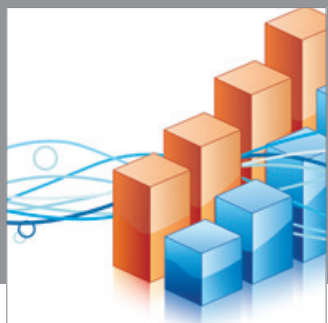

Advances in

Operations Research

mansans

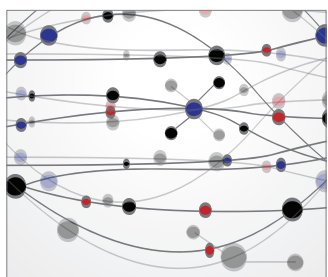

The Scientific World Journal
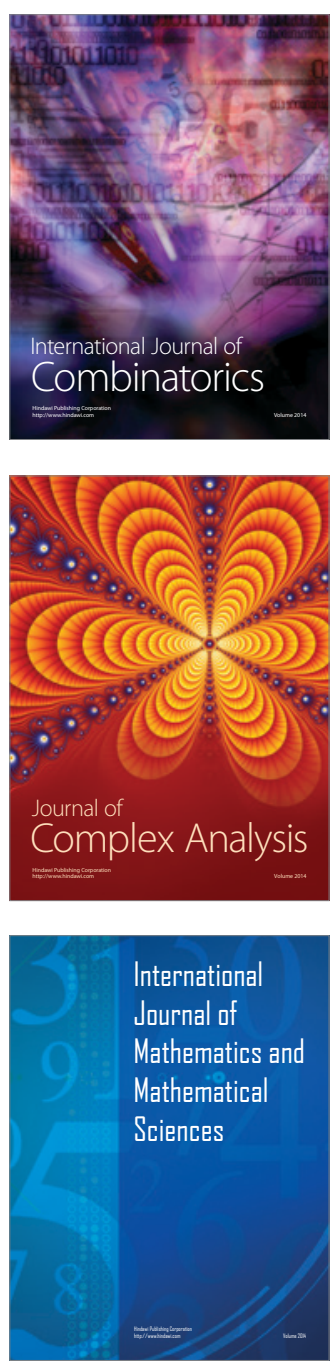
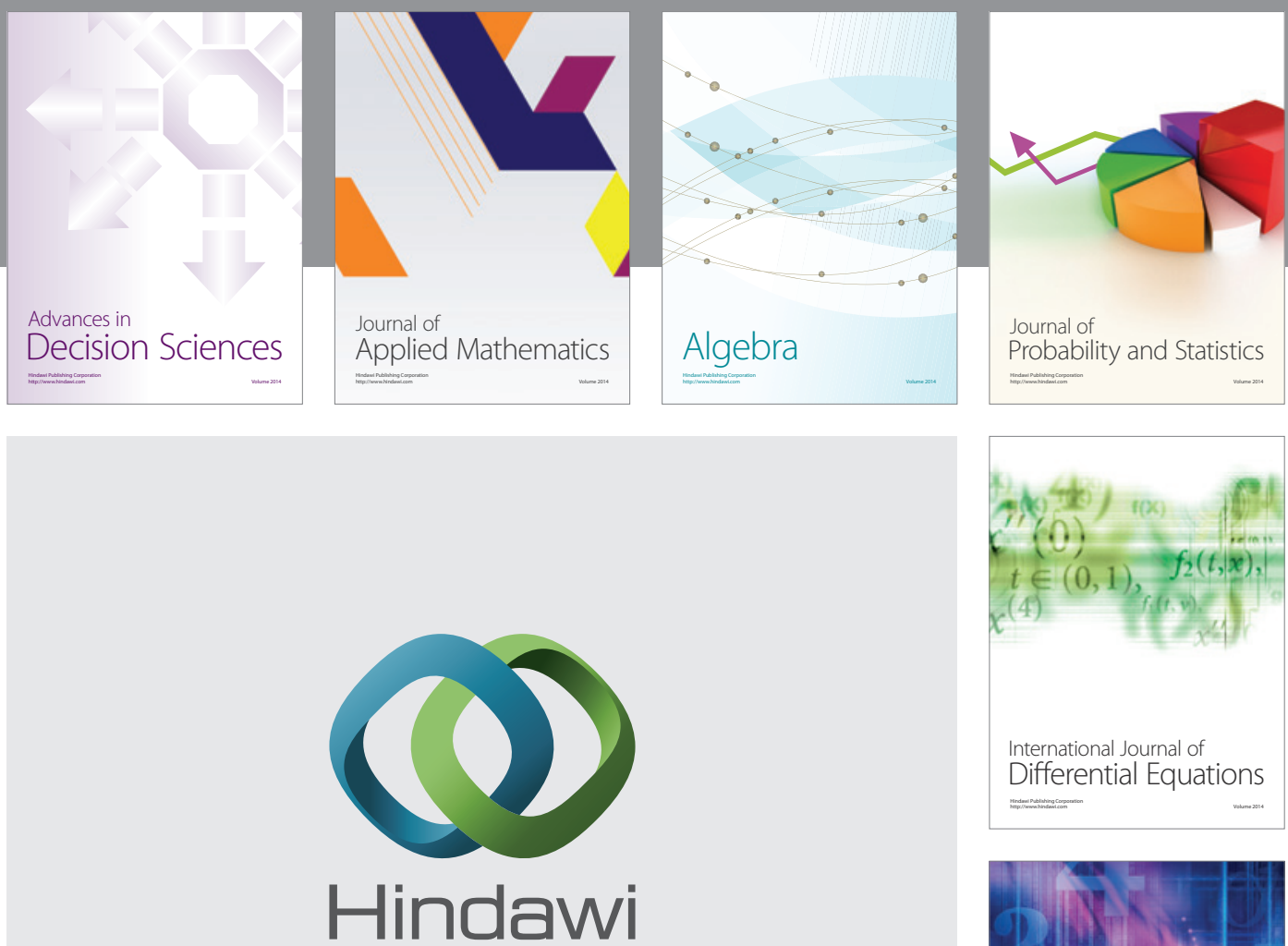

Submit your manuscripts at http://www.hindawi.com
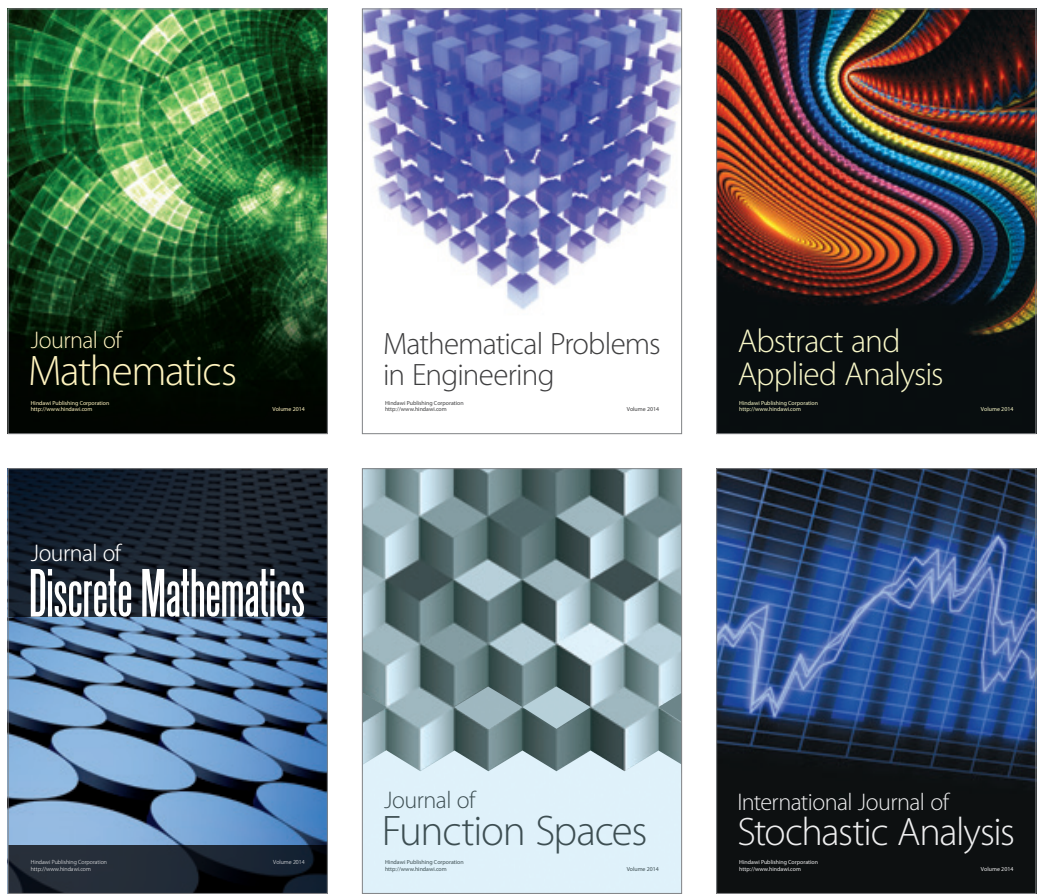

Journal of

Function Spaces

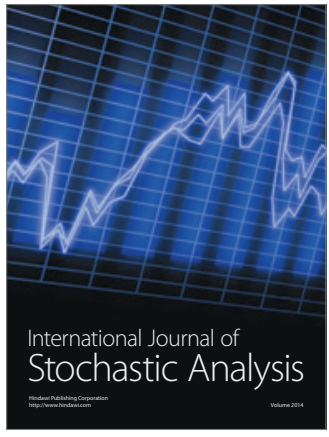

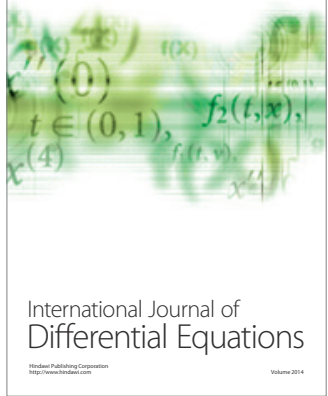
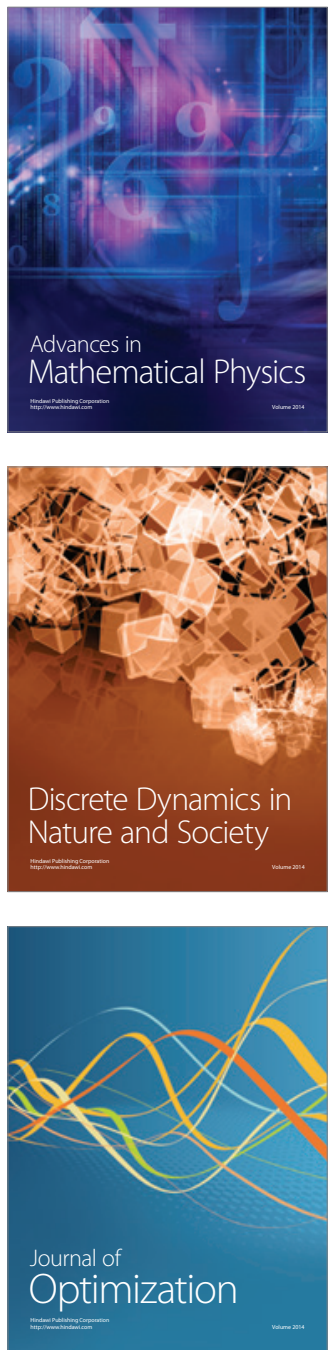